\title{
Picosatélite diseñado para medir concentración de gases de efecto invernadero y registrar imágenes en vuelo
}

| Fecha de recibido: 18 de febrero del 2021 | Fecha de aprobación: 12 de abril del 2021 |

\section{Juan David Osorio Henao \\ Ingeniero civil}

Estudiante de Ingeniería Aeroespacia de la Universidad de Antioquia

Colombia

Grupo de investigación Aerospace Science and Technology ReseArch (ASTRA)

Semillero de investigación Voyage

Función de investigador: teórico experimental y escritura

https://orcid.org/0000-0003-4166-9504

$\triangle$ juan.osorioh1@udea.edu.co

\section{Melissa}

Galeano Ruiz

Estudiante de Ingeniería Mecánica

Universidad de Antioquia Colombia

Grupo de investigación Aerospace Science and Technology ReseArch (ASTRA)

Semillero de investigación Voyage

Función de investigador: teórico y escritura https://orcid.org/0000-0003-2396-5721

$\bowtie$ melissa.galeanor@udea.edu.co

\section{Johan Jabrini \\ Botina Monsalve}

Estudiante de Ingeniería Aeroespacial

Universidad de Antioquia Colombia

Grupo de investigación Aerospace Science and Technology ReseArch (ASTRA)

Semillero de investigación Voyager

Función de investigador: teórico y escritura https://orcid.org/0000-0003-0337-844X

\ jabrini.botina@udea.edu.co

\section{Juan Pablo}

Higuita Echavarría

Estudiante de Ingeniería Mecánica

Universidad de Antioquia

Colombia

Grupo de investigación Aerospace Science and Technology ReseArch (ASTRA)

Semillero de investigación Voyager

Función de investigador: teórico y escritura https://orcid.org/0000-0003-2545-2305

凹jpablo.higuita@udea.edu.co
David Andrés

Díaz Álvarez

Estudiante de Ingeniería Aeroespacial

Universidad de Antioquia Colombia

Grupo de investigación Aerospace Science and Technology ReseArch (ASTRA)

Semillero de investigación Voyager Función de investigador: teórico, experimental y escritura

https://orcid.org/0000-0002-3487-6735 \david.diaza@udea.edu.co

\section{Luis Felipe}

Burbano Mosquera

Estudiante de Ingeniería Aeroespacial

Universidad de Antioquia Colombia

Grupo de investigación Aerospace Science and Technology ReseArch (ASTRA)

Semillero de investigación Voyager Función de investigador: teórico y escritura https://orcid.org/0000-0003-1748-8631 $\bowtie$ felipe.burbano@udea.edu.co

Cómo citar este artículo: Osorio Henao, J. D., Botina Monsalve, J. J., Díaz Álvarez, D. A., Galeano Ruiz, M., Higuita Echavarría,

J. P., \& Burbano Mosquera, L. F. (2021). Picosatélite diseñado para medir concentración de gases de efecto invernadero y registrar imágenes en vuelo. Ciencia y Poder Aéreo, 16(1), 75-86, https://doi.org/10.18667/cienciaypoderaereo.697 


\section{Picosatélite diseñado para medir concentración de gases de efecto invernadero $y$ registrar imágenes en vuelo}

Resumen: El equipo VoltaX, conformado por estudiantes del programa de Ingeniería Aeroespacial de la Universidad de Antioquia, desarrolló un CanSat - sistema satelital del tamaño de una lata de refresco- para la competencia Retos de Innovación CanSat Colombia 2020, evento organizado por la Sociedad de Sistemas Electrónicos y Aeroespaciales (AESS por sus siglas en inglés) del capítulo colombiano del Instituto de Ingenieros Eléctricos y Electrónicos (IEEE). El satélite fue diseñado para cumplir con los requerimientos de misión dados por la organización, los cuales incluían la transmisión constante de los datos a la estación terrena a 1000 m de distancia, la medición de temperatura, la aceleración lineal, los campos magnéticos y la presión atmosférica para determinar la altitud. Adicionalmente, se utilizó un sensor de gas para la medición de metano y una cámara GoPro para tomar imágenes de alta calidad, con el fin de hacer un monitoreo de los gases de efecto invernadero y utilizar las imágenes aplicadas a la agricultura de precisión. En la fabricación del prototipo se emplearon la manufactura aditiva y los componentes electrónicos Commercial Off-The-Shelf (cots por sus siglas en inglés, que traduce "productos comerciales estándar") para minimizar los costos. Por último, se realizaron pruebas funcionales de todos los subsistemas, con las cuales se corroboró el correcto funcionamiento del CanSat. Cabe agregar que el picosatélite, identificado con las placas VOLT4201, consiguió en la competencia el segundo puesto en su categoría.

Palabras clave: CanSat; producto comercial estándar (COTs por sus siglas en inglés); gases de efecto invernadero (GEI); unidad de medición inercial (IMU por sus siglas en inglés); telemetría; sensoramiento remoto; sistema de posicionamiento global (GPS por sus siglas en inglés).

Abstract: The VoltaX team, made up of Aerospace Engineering students at the University of Antioquia, developed a CanSat - a satellite system the size of a soda can - for the CanSat Colombia 2020 Innovation Challenge, an event organized by the IEEE Aerospace and Electronic Systems Society (AESS) Colombian Chapter. The satellite was designed to meet the mission requirements established by the organization, which included the constant transmission of data to the earth station at 1,000 m distance, measuring variables such as temperature, linear acceleration, magnetic field, and atmospheric pressure. Additionally, a gas sensor and a GoPro camera were used to estimate methane concentrations and capture high-quality images, respectively, with the purpose of monitoring greenhouse gases and using the images for precision agriculture applications. Additive manufacturing and commercial-off-the-shelf (coTs) electronic components were used to build the prototype, thus minimizing costs. Finally, functional tests were carried out on all the subsystems, confirming the correct operation of the CanSat. The designed picosatellite, identified with volT4201 plates, took the second place within its category.

Keywords: CanSat; commercial off-the-shelf (COTS); greenhouse gas; inertial measurement unit (IMU); telemetry; remote sensing; Global Positioning System (GPS).

Resumo: A equipe VoltaX, formada por estudantes do programa de Engenharia Aeroespacial da Universidade de Antioquia, desenvolveu um CanSat -sistema por satélite do tamanho de uma lata de refrigerante- para a competição Desafios de Inovação CanSat Colômbia 2020, evento organizado pela Sociedade de Sistemas Eletrônicos e Aeroespaciais (AESS) do capítulo colombiano do IEEE (Instituto de Engenheiros Elétricos e Eletrônicos). O satélite foi desenhado para cumprir com os requerimentos de missão dados pela organização, os quais incluíam a transmissão constante dos dados à estação terrena a $1000 \mathrm{~m}$ de distância, a medição da temperatura, a aceleração linear, os campos magnéticos e a pressão atmosférica para determinar a altitude. Além disso, um sensor de gás para a medição de metano e uma câmera GoPro foram usados para tirar imagens de alta qualidade, a fim de monitorar os gases do efeito estufa e usar as imagens aplicadas à agricultura de precisão. Na fabricação do protótipo foram empregados a manufatura aditiva e os componentes eletrônicos Commercial Off-The-Shelf (cots por suas siglas em inglês, que traduz "produtos comerciais padrão") para minimizar os custos. Por último, foram realizados testes funcionais a todos os subsistemas, com os quais se corroborou o bom funcionamento do CanSat. Deve-se acrescentar que o picosatélite, identificado com as placas VOLT4201, conseguiu na competição o segundo lugar na sua categoria.

Palavras-chave: CanSat; produto comercial padrão (cots por sua sigla em inglês); Gases do Efeito Estufa (GEE); Unidade de Medição Inercial (IMU por sua sigla em inglês); Telemetria; Sensoriamento remoto; Sistema de Posicionamento Global (GPS por sua sigla em inglês) 
En la actualidad, acercarse al desarrollo espacial es un gran reto para los países en crecimiento, debido a que suele ser costoso; sin embargo, con la evolución de la electrónica muchos componentes han presentado una disminución en su costo en las últimas décadas (Fernández \& Medel, 2019). Gracias a esto han nacido varias propuestas educativas que utilizan la tecnología de bajo costo conocida como producto comercial estándar (COTS), y que pretende desarrollar picosatélites como los CanSat (Paulo Silva Neto et al., 2019). El CanSat es un dispositivo electromecánico que trata de simular los subsistemas de un satélite real en las dimensiones aproximadas de una lata de refresco (Anchino, 2019). El nacimiento de este tipo de propuestas ha hecho que países como México, Brasil y Colombia comiencen a implementar este modelo educativo para estimular el desarrollo de tecnología aeroespacial en sus territorios. Un ejemplo de ello es el Cubedesign, una competencia latinoamericana de distintas universidades realizada en Brasil y que ha tenido una gran divulgación y crecimiento en los últimos años (INPE, 2020).

En Colombia existe el concurso de diseño de CanSat, Reto Innovación CanSat, organizado por el capítulo colombiano de la AESS para grupos de estudiantes. En esta competencia se desarrolló el CanSat VOLT4201 del equipo Voltax de la Universidad de Antioquia. Este grupo compitió en la categoría Cóndores, compuesta por estudiantes de primero a séptimo semestre de diferentes universidades colombianas, y logró ser finalista con el segundo puesto (AESS, 2020). En los lineamientos del concurso se asignaron las misiones Sabio Caldas y Nicola Tesla, las cuales determinaron los requerimientos para el diseño y construcción del CanSat; el proceso de diseño, pruebas, integración, manufactura y resultados se describen a continuación.

\section{Objetivo de la misión}

Actualmente es necesario medir el impacto de algunas actividades en el cambio climático, por lo que es importante medir los gases de efecto invernadero que producen y tienen mayor repercusión en la atmósfera; teniendo esto en cuenta, el primer objetivo del CanSat es servir como plataforma que provea información de la concentración de gases de efecto invernadero, en específico el metano, en donde se desea realizar el lanzamiento (Chaudhry \& Mishra, 2015; Faroukh et al., 2019). Por este motivo, se usa un sensor de gas MQ2 para medir la concentración de metano en la atmósfera.

Cabe aclarar que el equipo está ubicado en el oriente antioqueño, por lo cual desde el principio se planteó tener un monitoreo de los gases producto de las actividades de esta zona. Según un estudio realizado por la Corporación Autónoma Regional de las Cuencas de los Ríos Negro y Nare (Cornare) la ganadería representa el $40 \%$ de la actividad económica de la zona (Cornare, 2016); por lo anterior, es importante saber cómo esta actividad contribuye al cambio climático (Constantini et al., 2018). Además, el CanSat posee una cámara GoPro mediante la cual se obtienen imágenes a lo largo del vuelo, que pueden ser utilizadas para aplicaciones agrícolas, territoriales o topográficas; por esta razón, el CanSat está orientado a ser una herramienta en el sector agrícola e incursionar en lo que se conoce como agricultura de precisión.

Además, utilizar la cámara permite detectar ciertos índices vegetales "como el Triangular Greenness Index (TGI)" para medir la cobertura foliar de un cultivo (Hunt et al., 2012) y determinar su salud. En resumen, los objetivos de la misión son medir la concentración de gases de efecto invernadero, capturar imágenes que puedan ser usadas en la agricultura de precisión y, por último, transmitir los datos a una estación terrena (Fretes \& Gómez, 2018).

\section{Requerimientos de la misión}

Los requerimientos dados por el concurso se resumen en la tabla 1. El diseño fue trabajado a partir de estos y se logró alcanzar satisfactoriamente las exigencias en cada etapa. 
Tabla 1.

Requerimientos de la misión dados

por la organización del concurso

\section{Requerimientos}

\begin{tabular}{l|l} 
Funcionales & Medir temperatura, presión atmosférica y altitud. \\
\cline { 2 - 2 } Operacionales & $\begin{array}{l}\text { Añadir sensores de aceleración, geolocalización, } \\
\text { giroscopio, altímetro, telecomandos, la medición de } \\
\text { gases y la toma de imágenes. }\end{array}$ \\
$\qquad$ & $\begin{array}{l}\text { Transmisión de datos una vez por segundo } \\
\text { a la estación terrena. }\end{array}$ \\
\cline { 2 - 2 } Almacenamiento a bordo. \\
\cline { 2 - 2 } Restricciones & $\begin{array}{l}\text { Mostrar evidencias de captura y recepción de datos } \\
\text { mediante gráficos de tiempo-altitud, temperatura- } \\
\text { altitud, presión atmosférica-altitud y otros. }\end{array}$ \\
\cline { 2 - 2 } & $\begin{array}{l}\text { Alcance mayor a } 1 \text { km, entre el CanSat } \\
\text { y la estación terrena. }\end{array}$ \\
\cline { 2 - 2 } Peso máximo de $500 \mathrm{~g}$. \\
\cline { 2 - 2 } $\begin{array}{l}\text { Todos los componentes deben estar contenidos en } \\
\text { la lata, los únicos componentes externos permitidos } \\
\text { son el paracaídas y la antena. }\end{array}$ \\
\cline { 2 - 2 } $\begin{array}{l}\text { Dimensiones de una lata de } 350 \mathrm{ml} \\
\text { (geometría cilíndrica). }\end{array}$ \\
\hline
\end{tabular}

Fuente: AESS (2020).

\section{Subsistemas}

\section{Computadora de vuelo}

La computadora de vuelo se encarga de la medición de variables físicas, control de componentes, almacenamiento y comunicación de los datos, además, envía señales a diversos componentes que posee el CanSat, regula los intervalos de tiempo en los que se realizarán las actividades y controla tareas de los demás subsistemas. Por lo tanto, controla los sensores de la Unidad de Medición Inercial (IMU, por sus siglas en inglés), con la que se puede obtener información directamente relacionada con el vuelo, como la posición, orientación, aceleración, velocidad y presión; así mismo, es responsable de suministrar energía a otros componentes como el LoRa Ra-02, utilizado en el subsistema de telemetría.

Para la selección de la computadora de vuelo, se analizaron diferentes alternativas como Arduino Uno, Arduino Nano, Raspberry Pi, Arduino Mega Pro Mini, entre otras. Se eligió el Arduino Mega Pro debido a aspectos como el precio, el tamaño, la compatibilidad de protocolos de comunicación que posee I2C y SPI, número de pines digitales (54), entradas analógicas (16), salida de voltaje a $5 \mathrm{~V}$ y a 3,3 $\mathrm{V}$, velocidad de reloj (16 MHz), facilidad de programación y compatibilidad con múltiples componentes electrónicos.

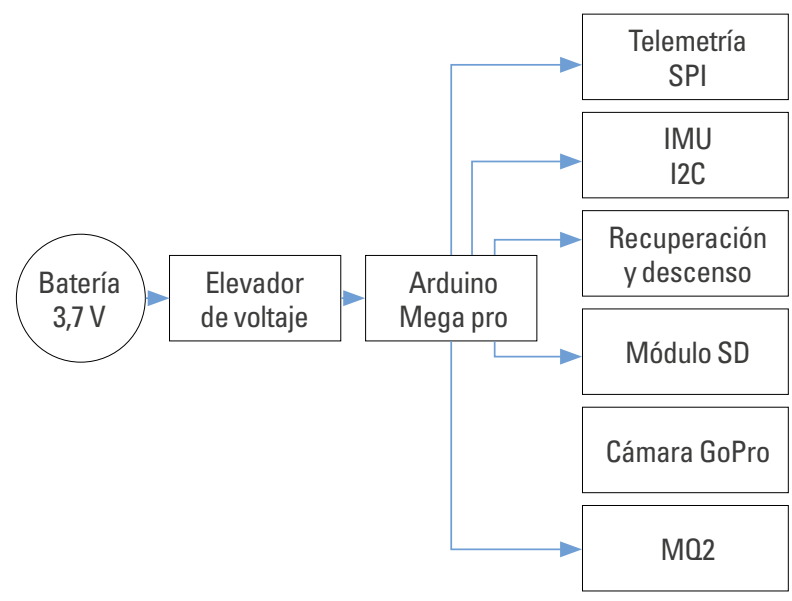

Figura 1.Diagrama de los componentes electrónicos usados en el CanSat y su suministro de voltaje.

Fuente: elaboración propia.

En la selección de la ıMU se contemplaron diversas posibilidades, entre estas GY-89, GY-52 y GY-802; sin embargo, se notó que todas las alternativas tenían similitud en cuanto al voltaje de funcionamiento, los protocolos de comunicación (I2C y SPI), la sensibilidad y demás parámetros, así que se optó por elegir la opción más económica, en este caso la IMU GY-89, que es una combinación de los sensores BMP180 (barómetro), L3GD320 (giróscopo) y LSM303D (magnetómetro). El diagrama de la computadora de vuelo se puede ver en la figura 1.

También se decidió emplear un módulo SD para el almacenamiento de datos durante el vuelo. Este no es el sistema principal de almacenamiento de datos, ya que se generó el envío de datos cada segundo a la estación terrena, por lo que el módulo SD es un sistema de respaldo a la transmisión de datos; en este caso, la misma información que se envía a la estación terrena es almacenada en una SD de $8 \mathrm{~GB}$, un componente redundante para garantizar la recopilación de los datos durante el vuelo. 
Adicional a los sensores mencionados, se empleó un sensor MQ2, que es electroquímico, el cual genera una resistencia de acuerdo con la concentración de gases que hay en el exterior. Para utilizar este sensor, se requiere realizar un calentamiento previo de diez minutos, el rango que puede medir este sensor es de 300 a 10.000 ppm de metano, propano, butano, alcohol, hidrógeno y otros, y el voltaje que requiere es de $5 \mathrm{~V}$-revisar la figura 2- (Heyasa \& Galarpe, 2017).

Para la programación del subsistema, se usaron diferentes librerías de Arduino que permiten interactuar con los sensores instalados en el CanSat. Así mismo, como se mencionó, para la medición del gas metano se utilizó un sensor MQ2, y para la IMU GY-89 se usaron librerías de tres sensores: LSM303D (Herrada, 2020b), L3GD20 (Herrada, 2020a) y BMP180 (Ladyada, 2020).

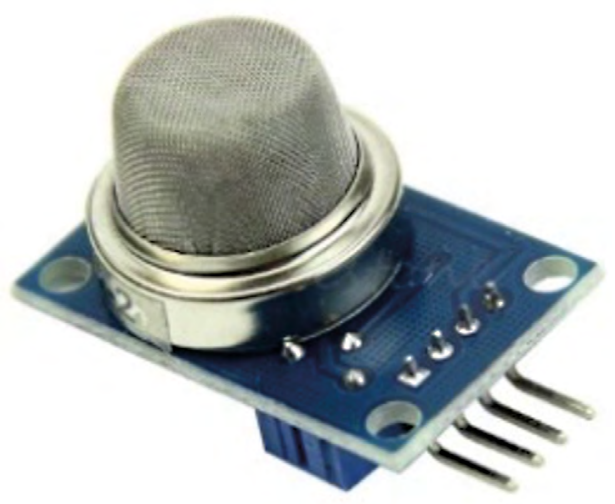

Figura 2. Sensor M02.

Fuente: imagen tomada de Seed (2015).

\section{Estructura}

La estructura es la encargada de albergar todos los componentes electrónicos del CanSat, la cual brinda soporte y protección, además de desempeñar un papel fundamental para la organización y distribución de los demás subsistemas, por lo que es un punto clave para el éxito de la misión. Asimismo, la estructura se ve sometida a diferentes fuerzas producidas por las múltiples aceleraciones y vibraciones sufridas durante el vuelo (Colin, 2016). Por lo anterior, el equipo se enfocó en presentar una alternativa que minimizara estas adversidades, para garantizar un buen comportamiento frente a estos esfuerzos presentes en la estructura. La propuesta se centró en un diseño de geometría cilíndrica similar a una lata de refresco de $350 \mathrm{ml}$, con un armazón interior, de acuerdo con una distribución modular de tipo estante (Méndez, 2017), donde se organizan los diferentes componentes electrónicos de cada subsistema en distintas placas horizontales dispuestas a lo largo del CanSat; estas placas son sostenidas y acopladas entre sí por una serie de columnas verticales que se encargan de soportar la mayoría de los esfuerzos provenientes del vuelo y aterrizaje.

La estructura descrita, así como su distribución, se presentan en la figura 3 , en esta se pueden observar los componentes estructurales principales:

1. Una carcasa exterior que sigue un patrón de lámina delgada formando una sección tubular con dos tapas en sus extremos, adicionalmente cuenta con diferentes aberturas necesarias para los dispositivos encargados del análisis de gases en la atmósfera y de la captura de imágenes del terreno. Su función principal es proteger los dispositivos electrónicos y la estructura interna de impactos, además de aislarlos del medio externo. A su vez, se decidió fabricar esta carcasa en Ácido Poliláctico (PLA), que permite ser manufacturado por impresión 3D, además posee buenas propiedades mecánicas, baja densidad y es fácil de adquirir.

2. Tres placas horizontales que siguen un patrón circular conforman la base para alojar los dispositivos electrónicos y sirven como punto de anclaje a otros componentes estructurales, como las tres columnas ubicadas entre las dos placas superiores y el sistema de cuatro columnas inferiores diseñado exclusivamente para soportar el módulo de captura de imágenes. Ambos sistemas están construidos en nailon 6,6 y se encargan de reducir las vibraciones y la mayoría de los esfuerzos provenientes del vuelo y aterrizaje. 


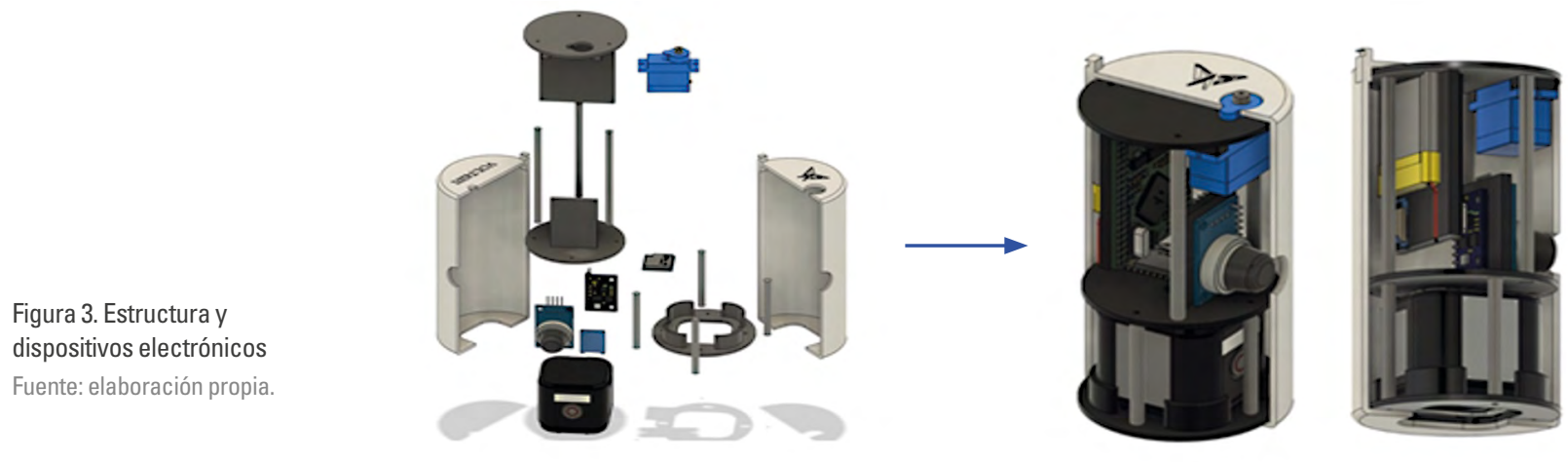

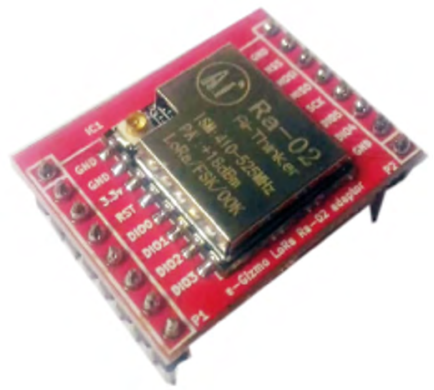

Figura 4. Módulo Lo-Ra 02, imagen tomada de e-Gizmo Fuente: elaboración propia.

\section{Comunicaciones y telemetría}

El subsistema de telemetría se encarga de recolectar y transmitir datos en tiempo real durante todas las fases de vuelo desde el picosatélite hasta la estación terrena, la cual es capaz de almacenar esta información de manera ordenada para ser analizada posteriormente, según la misión especificada. La interacción entre el CanSat y el usuario se da a partir de este subsistema que posee dos partes primordiales: un módulo transmisor que toma la información obtenida por la carga útil durante el vuelo y la transmite en forma de ondas de radio, y un módulo receptor en tierra conectado a un computador a través de un módulo Arduino, en donde se almacena y procesa la información.

En el sistema de comunicación se emplearon dos módulos LoRa Ra-02 -figura 4- debido a su alto rango de transmisión, el cual es de $10 \mathrm{~km}$, suficiente para la misión que exige un rango de $1 \mathrm{~km}$. Además, tiene una tasa de transmisión de datos $(300 \mathrm{~kb} / \mathrm{s}$ ) y su precio es asequible (e-Gizmo, 2017).

En la figura 5 se muestra el esquema del proceso de comunicación desde el CanSat hacia la estación terrena, allí el Arduino recibe los datos enviados por el módulo transmisor. Este módulo los envía a la estación terrena, en donde son recibidos por el módulo receptor, después se transmiten al microcontrolador que a su vez está conectado a una computadora en donde los datos son visualizados usando un código escrito en Python.
Figura 5. Proceso de la comunicación y la información Fuente: elaboración propia.

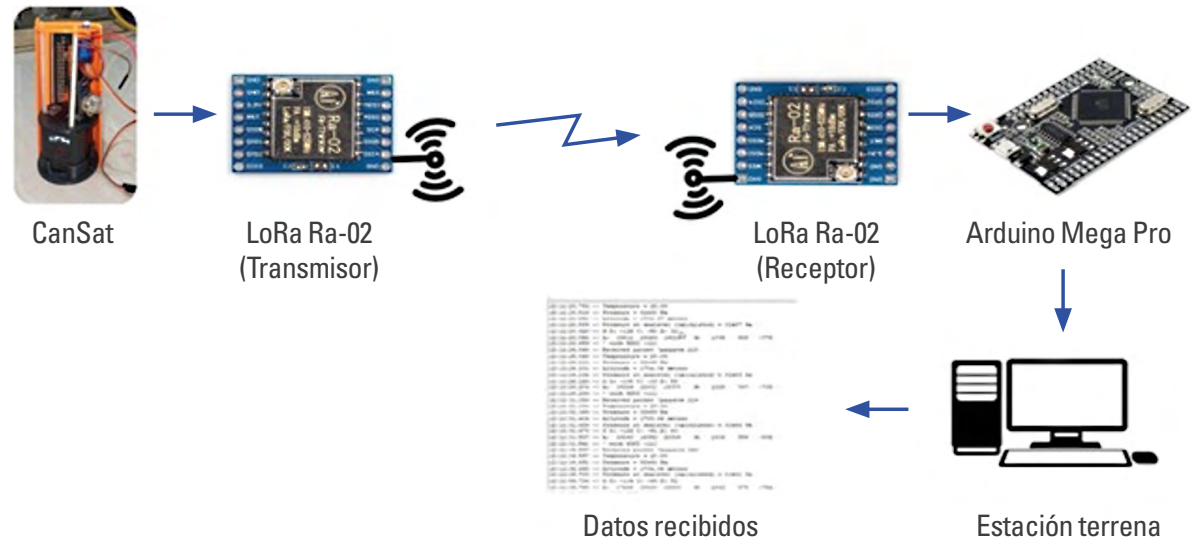




\section{Descenso y recuperación}

Es fundamental que el CanSat llegue a tierra sin ningún daño, conservando sus componentes intactos, y que transmita señales que permitan ubicar su lugar de aterrizaje; de esta forma se posibilita una futura reutilización del picosatélite y un cumplimiento idóneo de la misión, de lo cual se encarga el subsistema de recuperación.

El componente principal del subsistema de recuperación es un paracaídas hecho en ripstop nailon, este material es confiable por su alta resistencia a la tracción. A partir del artículo de Ferraz et al. (2017), en donde se explica el diseño de un CanSat y los requerimientos básicos de la competencia de picosatélites (CanSat Competition, 2020), se desarrollaron los cálculos necesarios para el descenso y se encontró que lo ideal sería una velocidad de descenso de $5 \mathrm{~m} / \mathrm{s}$, para que los instrumentos del CanSat no sufran ningún daño durante el aterrizaje. En ese sentido, la geometría elegida para el paracaídas fue de tipo hexagonal, con un diámetro de $60 \mathrm{~cm}$ y con seis cuerdas sujetas al CanSat, una en cada esquina del hexágono; con esta configuración se esperó tener un tiempo de descenso aproximado de $200 \mathrm{~s}$ desde una altura de $1000 \mathrm{~m}$. En la tabla 2 se pueden ver las velocidades de llegada del CanSat desde tres alturas distintas, estas fueron simuladas en el programa Open Rocket (ESA, n. d; Mohd et al., 2020).

Para la activación del paracaídas se realizó un algoritmo que determina la variación de su posición cada segundo con respecto a la medición anterior; en caso de que aumentara su altitud, el subsistema permanecía sin activarse. No obstante, tan pronto este empieza a obtener cambios de altitud negativos, el Arduino envía una señal eléctrica al servomotor, lo cual activa el subsistema de recuperación. El accionamiento del paracaídas se hace con la ayuda de un servomotor que al actuar suelta una banda elástica que está sujeta al paracaídas. Además, el picosatélite tiene integrado un módulo Sistema de Posicionamiento Global (GPS, por sus siglas en inglés), que transmite su posición a la estación terrena y facilita la recuperación del CanSat.
Tabla 2.

Simulaciones de descenso

\begin{tabular}{l|c|c|c} 
& $\begin{array}{c}\text { Velocidad } \\
\text { del viento }(\mathbf{m} / \mathbf{s})\end{array}$ & $\begin{array}{c}\text { Altura en que } \\
\text { se abre el } \\
\text { paracaídas }(\mathbf{m})\end{array}$ & $\begin{array}{c}\text { Velocidad } \\
\text { de llegada } \\
\text { al suelo (m/s) }\end{array}$ \\
\hline Simulación 1 & 0 & 480 & 5,6 \\
\hline Simulación 1 & 30 & 338 & 5,5 \\
\hline Simulación 2 & 0 & 557 & 4,9 \\
\hline Simulación 2 & 30 & 497 & 6,5 \\
\hline Simulación 3 & 0 & 1061 & 4,3 \\
\hline Simulación 3 & 30 & 1042 & 5,3 \\
\hline
\end{tabular}

Fuente: elaboración propia.

Por último, se usó el software Open Rocket para calcular la altura con relación al tiempo -figura 6usando los datos de la simulación 3 con la velocidad del viento de $0 \mathrm{~m} / \mathrm{s}$-tabla $2-$. El eje vertical es la altitud dada en metros, el eje horizontal es el tiempo dado en segundos, y la línea azul es la altitud que varía con respecto al tiempo, desde el momento en que se activa el paracaídas (1061 m) hasta que el CanSat aterriza.

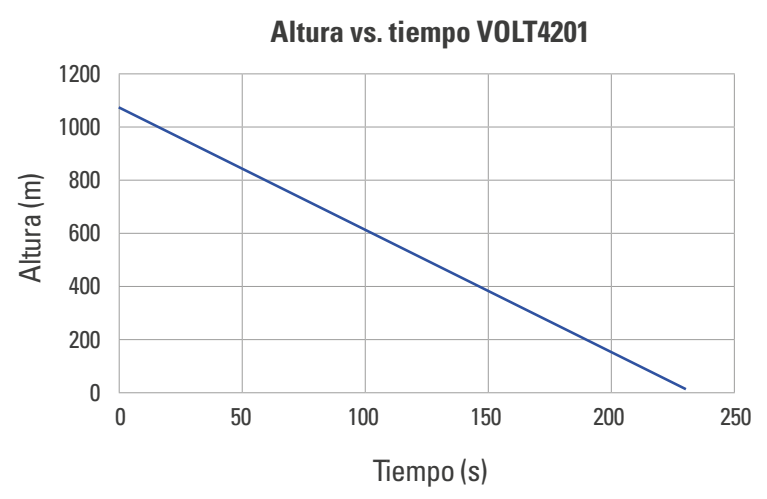

Figura 6. Gráfica de la simulación del descenso altura vs. tiempo realizada en 0 pen Rocket Fuente: elaboración propia.

\section{Potencia eléctrica}

Es el subsistema que provee la energía a los componentes electrónicos presentes en el CanSat a excepción de la cámara, porque esta posee su batería propia. Está compuesto esencialmente por una 
batería que suministra la energía necesaria para los demás subsistemas y componentes. En este caso, el Arduino requiere una alimentación entre $7 \mathrm{~V}$ y $9 \mathrm{~V}$, los demás componentes del CanSat se comunican mediante los puertos I/O que posee el Arduino; además, este tiene pines a tierra de $3,3 \mathrm{~V}$ y $5 \mathrm{~V}$ que permiten la adecuada conexión de los otros componentes, de acuerdo con sus especificaciones técnicas. De este modo, el Arduino actúa como un sistema de distribución de energía y sirve como controlador de todos los componentes.

Al contar con una batería propia, la cámara no necesita alimentación de la batería que provee energía al Arduino. Ahora bien, los criterios de selección para la batería son el voltaje que posee, la capacidad de carga y descarga, la facilidad de ensamblaje, el tamaño, peso y la facilidad de carga. Teniendo en cuenta lo anterior se optó por el uso de una batería de LiPo de 3,7 V de 1200 mAh - figura 7-. Este tipo de batería fue seleccionada debido a su gran capacidad de descarga, necesaria para alimentar eficazmente el circuito de activación del sistema de descenso, ya que suele requerir alta corriente en un intervalo de tiempo muy corto, además, la energía que almacena es suficiente para la alimentación de los demás componentes electrónicos por el tiempo que se requiere. No obstante, debido a su voltaje limitado, requiere de un elevador de voltaje para entregar los valores requeridos por otros componentes. Su bajo voltaje se debe a que las baterías tipo LiPo, por lo general, son usadas en drones y cuentan con un voltaje de salida de $3,7 \mathrm{~V}$, baterías de mayor voltaje suelen ser de mayor tamaño y peso, por lo que se descarta esta opción; estas baterías tienen una tecnología de mayor duración que permite aumentar los ciclos de carga y descarga, por lo que ambientalmente tiene un menor impacto. Adicionalmente, se requiere de un elevador de voltaje para llegar al voltaje que necesita el Arduino, cuyo rango de operación está entre $7 \mathrm{~V}$ y $9 \mathrm{~V}$.

\section{Integración y manufactura}

Es necesario integrar todos los subsistemas y lograr que trabajen en armonía para un correcto desarrollo de la misión, lo cual se realiza al momento de hacer la manufactura del prototipo. La manufactura está caracterizada por la implementación de tecnologías de prototipado, un ejemplo de ello es la impresión 3D por medio de software especializado que le permitió al equipo afrontar de forma remota todas las dificultades de construcción y diseño que se pudieran presentar durante el periodo de contingencia debido al virus SARS-CoV-2 (Ministerio de Salud de Colombia, 2020).

La estructura fue fabricada en impresión 3D usando solamente PLA, con una forma cilíndrica hueca. A su

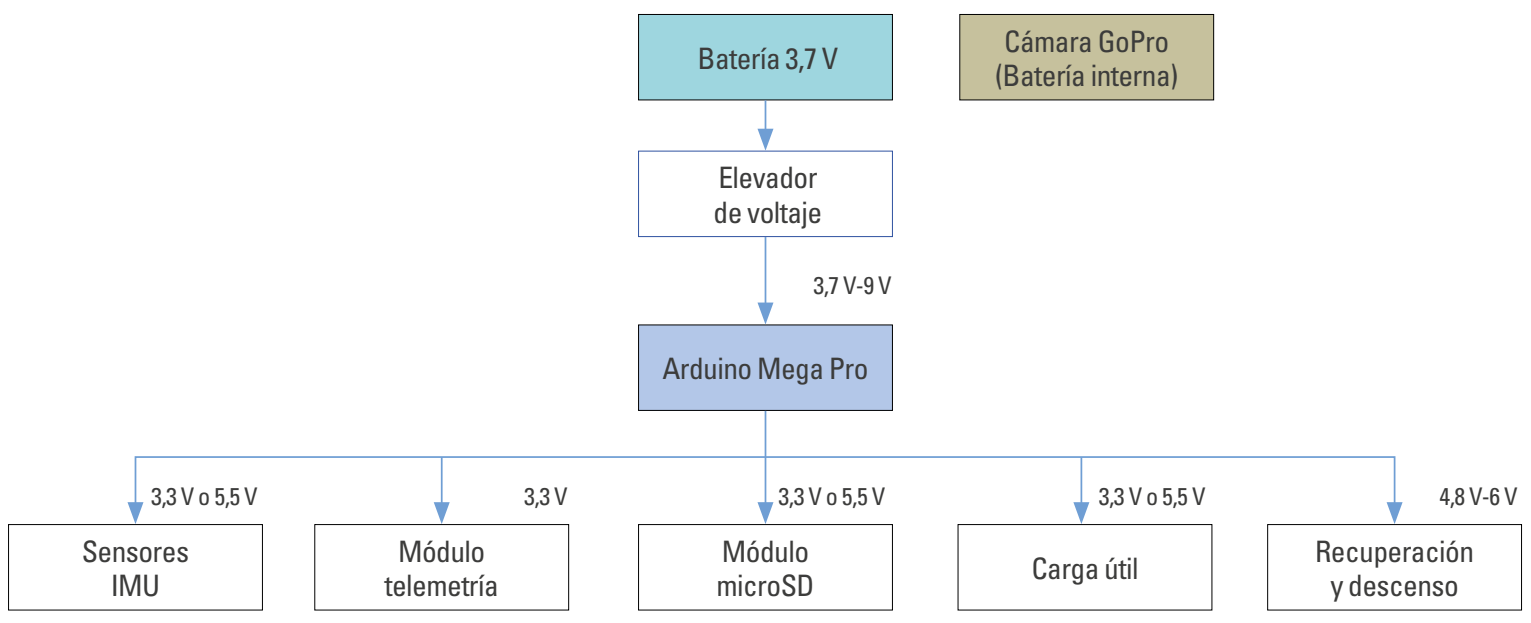

Figura 7. Esquema de distribución de voltaje

Fuente: elaboración propia. 
vez, posee dos tapas en sus extremos, las cuales se apoyan en tres columnas verticales de nailon y tienen tres niveles horizontales, también impresos en 3D, en los cuales se soporta la electrónica del CanSat. La electrónica se dispone en los tres niveles - como ya se describió-, los subsistemas de telemetría, potencia y computadora de vuelo se prueban conjuntamente a medida que se ensamblan, para corroborar el correcto funcionamiento de sus instrumentos, dado que todos dependen entre sí.

Después de verificar la correcta sujeción de cada elemento, se procedió a colocar las cubiertas -en la figura 3 se puede observar la integración y distribución de componentes en el CanSat-, el paracaídas se sujetó con sus seis cuerdas al soporte colocado en la parte superior de la estructura; por último, se plegó y se ubicó encima de la tapa superior del CanSat, donde se colocó la banda elástica tanto en el paracaídas como en el servomotor, de esta manera se completó la integración y ensamblaje del CanSat VOLT4201.

\section{Resultados}

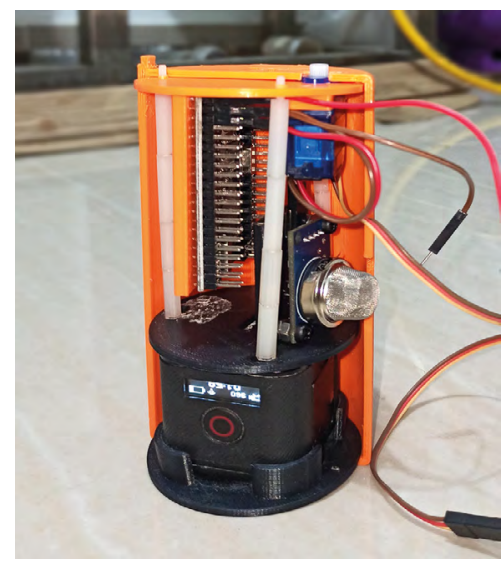

Figura 8. Prototipo CanSat VOLT4201

Fuente: elaboración propia.

En suma, se diseñó y prototipó un CanSat -figura 8- capaz de medir variables físicas relacionadas directamente con el vuelo, tales como velocidad, aceleración, posición, temperatura, presión, altitud (Heyasa \& Galarpe, 2017) y transmisión de la información en tiempo real a una estación terrena, que podría estar ubicada a una distancia mayor a $1000 \mathrm{~m}$. El sistema tiene, además, la capacidad de hacer captura de imágenes mediante una cámara GoPro. Por otra parte, el subsistema de recuperación es accionado en el apogeo mediante un servomotor, y libera el paracaídas que permite preservar los diversos componentes y aumentar el tiempo de vuelo logrando obtener una mayor cantidad de datos ambientales y de imágenes. El CanSat VOLT4201 es una herramienta de bajo costo, el valor de sus componentes fue de unos 330 USD -ver tabla 3 de peso y costo de los componentes-; es importante resaltar que el CanSat puede ser utilizado varias veces sin tener que comprar material extra.

Tabla 3.

Peso y costo de los componentes

\begin{tabular}{l|c|c}
\multicolumn{1}{c|}{ Componentes } & Peso (gramos) & Costo (COP) \\
\hline Arduino Mega Pro & 37 & 60,400 \\
\hline Cámara GoPro & 73 & 800,000 \\
\hline Servomotor & 9 & 5,399 \\
\hline Módulo LoRa Ra-02 & 2 & 28,000 \\
\hline Unidad de Medición Inercial (UMI) & 2 & 44,030 \\
\hline Batería & 30 & 75,000 \\
\hline Sensor de Metano MQ2 & 16 & 9,500 \\
\hline Paracaídas & 22 & 50,000 \\
\hline Módulo SD & 6 & 4,500 \\
\hline GPS & 14 & 30,000 \\
\hline Elevador de voltaje & 12 & 3,500 \\
\hline Antena LoRa & 20 & 13,200 \\
\hline Cables & 35 & 3,000 \\
\hline Estructura & 150 & 50,000 \\
\hline Peso total / Costo total & 429 & 1.176 .529 \\
\hline Fonte: laboraco propa &
\end{tabular}

Fuente: elaboración propia.

También se hicieron pruebas de toma de datos y telemetría para validar la programación, el diseño y el correcto funcionamiento de la electrónica presente en el CanSat; con ese objetivo se realizó el lanzamiento de varios cohetes fabricados por estudiantes de la Universidad de Antioquia y la Universidad Pontificia Bolivariana, en un ejercicio conjunto con la FAC desarrollado en el municipio de Puerto Boyacá (Fuerza Aérea Colombiana, 2019). 


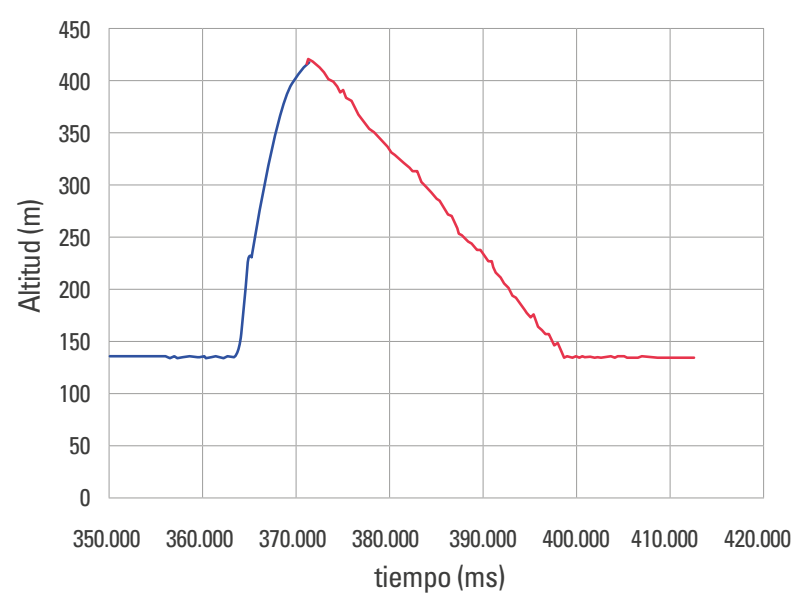

Figura 9. Perfil de vuelo obtenido en el lanzamiento en Puerto Boyacá Fuente: elaboración propia.

En el vuelo realizado se buscó obtener datos directamente relacionados con el vuelo, como altitud, aceleraciones, velocidades y presión -figura 9-; además, se obtuvo información de la concentración de gases en toda la trayectoria usando el sensor de gases MQ2. De esta manera fue posible elaborar la figura 11, donde se presenta la variación del voltaje. Es necesario tener en cuenta que el análisis de la variación de voltaje se asocia directamente con la cantidad de metano detectado, de acuerdo con su datasheet; sin embargo, hace falta realizar una calibración previa del sensor. Este resultado evidenció que el sensor funcionó correctamente durante el vuelo. Para realizar la calibración e interpretar los datos se debe calcular el $R_{0}$-resistencia inicial- en un ambiente donde se conozca la concentración de metano, y el $R_{s}$ que es la resistencia del sensor asociada con la concentración de determinado gas (Heyasa \& Galarpe, 2017). Para este proceso se deben utilizar las curvas que se aprecian en la figura 10. Sin embargo, no se obtuvieron los valores de concentración por la falta de un instrumento de referencia que permitiera calibrar el sensor y conseguir un dato más preciso de la concentración del metano en ppm.

Con el fin de probar el sistema de potencia antes de su integración en el CanSat se realizaron varias transmisiones de datos para analizar el tiempo de duración de batería y determinar la autonomía del CanSat. Los resultados mostraron que se podría tener una duración de hasta 23 minutos desde el encendido de la electrónica; después de ese momento empezó a fallar el envío de datos. Si se quisiera aumentar el tiempo de duración de la misión, se tendría que utilizar una batería diferente, sin embargo, el tiempo de vuelo que se planeó fue inferior a 4 minutos - de acuerdo con las simulaciones descritas-, por lo cual se considera que la batería de $1.200 \mathrm{mAh}$ tiene una capacidad de almacenamiento suficiente.

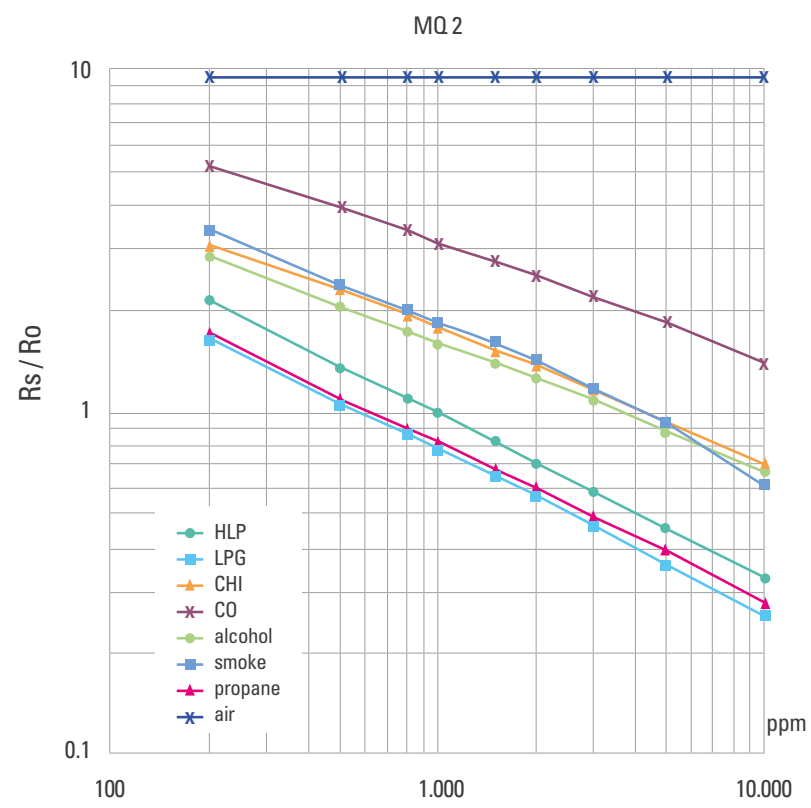

Figura 10. Relación $\frac{R_{0}}{R_{s}}$ para cada concentración de gas, obtenida a partir del datasheet del sensor M02

Fuente: elaboración propia.

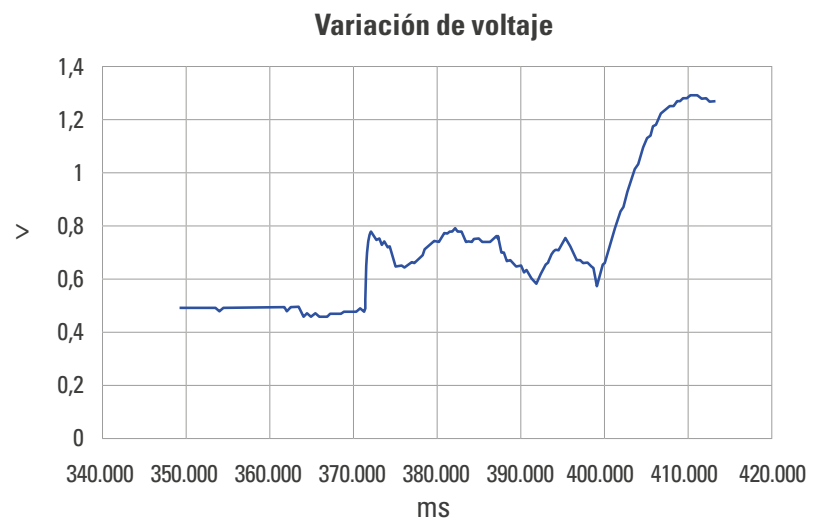

Figura 11. Variación de voltaje sensor M02 obtenido en el lanzamiento en Puerto Boyacá

Fuente: elaboración propia. 


\section{Conclusiones}

Se diseñó y prototipó un dispositivo CanSat de bajo costo que permite realizar mediciones relacionadas con el vuelo, como las aceleraciones y velocidades y, además, mide la concentración de gases en múltiples zonas de estudio. Asimismo, a partir de los datos obtenidos se puede comparar cómo cambia la concentración de gases a medida que varía la altitud y el uso del suelo, por lo que se podrían cuantificar los impactos que tienen diversas actividades económicas, transporte, industria, agricultura, entre otras.

El diseño y la construcción de un dispositivo de estas características en condiciones de aislamiento por las medidas de contingencia del virus SARS-CoV-2 implicaron un reto significativo para el equipo involucrado. No obstante, la utilización de tecnologías de trabajo remoto, el uso de software especializado para el diseño y las herramientas relacionadas con la transmisión, procesamiento y almacenamiento digitalizado de la información supuso una gran ventaja para el desarrollo de este proyecto, pues gracias a ello se alcanzaron los objetivos propuestos por el concurso y la misión, por lo cual cabe destacar la importancia de la implementación de este tipo de tecnologías en los futuros proyectos que enfrente el equipo.

Igualmente, la implementación de la cámara GoPro en el CanSat es una ventaja por su versatilidad al facilitar su programación e instalación; sus opciones de ángulo de visión permiten tener una alta calidad de imágenes o videos, adicionalmente, su capacidad de almacenamiento y batería es de buen rendimiento -aunque es el componente que tiene el mayor costo-, a largo plazo se vuelve una buena inversión.

La batería LiPo de 3,7 V de 1200 mAh funcionó correctamente como fuente de potencia, al igual que el Arduino Mega Pro, el cual trabajó adecuadamente como distribuidor de esta. Así mismo, el elevador de potencia fue completamente necesario para suministrar la energía suficiente al Arduino y cada componente.

Es necesario realizar pruebas de telemetría a mayores distancias, aunque los componentes usados en telemetría tienen una ficha técnica que en teoría funciona, es preciso evaluar el desempeño a grandes distancias, analizar el envío de datos y la calidad de la señal que se recibe. Así mismo, hay que tener en cuenta las vibraciones y posibles aceleraciones bruscas que se pueden generar en vuelo, para garantizar el suministro de información a la estación terrena de forma constante, luego se debe asegurar que los componentes no sean afectados por alguna perturbación del CanSat en su movimiento y que reciban energía constantemente.

Por último, el CanSat no ha sido probado en un ambiente real de trabajo, por lo que se espera realizar una misión en globo aerostático en la cual se prueben todos los subsistemas del CanSat para así demostrar su funcionalidad. El CanSat servirá como base para futuros proyectos no solo del equipo, sino también de la población estudiantil de la Universidad de Antioquia en general. Es necesario mencionar que para realizar pruebas en el espacio aéreo se contó con la autorización de la Aerocivil y la FAC para evitar posibles percances 0 accidentes.

\section{Agradecimientos}

Agradecemos a la Universidad de Antioquia y al Semillero de investigación Voyager, por su apoyo y aprobación para el desarrollo de este proyecto; al grupo de investigación ASTRA y Cipsela, por su acompañamiento; a los profesores Elías Montoya y Jorge Zamora, por brindarnos sus asesorías y conocimientos. Finalmente, se agradece la colaboración de Andrés Nieto y Walter Agudelo en la fabricación, el ensamblaje y pruebas del CanSat.

Declaración de conflicto de interés: Los autores no manifiestan conflictos de interés institucionales ni personales.

\section{Referencias}

AESS, A. \& E. S. S. (2020). Innovación CanSat Colombia 2020. http://ieee.udistrital.edu.co/cansat/index.html 
CanSat Competition. (2020). CanSat Competition Guide 2021. http://www.cansatcompetition.com/docs/CanSat_Mis sion_Guide_2021.pdf

Carrero Urbina, J. (2017). El espacio, futuro de la Fuerza Aérea Colombiana. Ciencia y Poder Aéreo, 12(1), 202-208.

Chaudhry, V., \& Mishra, I. (2015). Zenith: A Nano-Satellite for Atmospheric Monitoring. SAE Technical Papers, 2015-September (September). https://doi.org/10.4271/ 2015-01-2395

Constantini, A., Pérez, G., Busto, M., González, F., Cosentino, V., Romaniuk, R., \& Taboada, Mi. (2018). Emisiones de Gases de Efecto Invernadero en la Producción Ganadera. https://repositorio.inta.gob.ar/bitstream/hand le/20.500.12123/4389/INTA_CIRN_InstitutodeSuelos_ Costantini_A_Emisiones_gases_efecto_invernadero_ produccion_ganadera.pdf?sequence=1\&isAllowed=y

Colín, Á., Bermúdez Reyes, B., Encarnación Morrobel, G., Lira Ibarra, G., Rosales, Z., Ávalos De La Cruz, L., Villarreal Méndez, M., Mendoza Martínez, J., \& Álvarez, B. (2016). Construcción de un picosatélite cansat. Ciencia UANL, 81, 34-38. http://eprints.uanl.mx/11599/1/Documento6.pdf

Cornare. (2016). Actividad económica actual en el Oriente Antioqueño y perspectivas de crecimiento verde y desarrollo compatible con el clima.

e-Gizmo. (2017). LORA Module RA-02. https://www.e-gizmo. net/oc/kits documents/LORA Module RA-02 V.1/LORA rev2.pdf

ESA, E. S. A. (n.d.). ESA - Design your parachute - A Guide to Landing Your CanSat Safely | Teach with Space T10. https:// www.esa.int/Education/CanSat/Design_your_parachute_A_Guide_to_Landing_Your_CanSat_Safely_Teach_ with_Space_T10

Faroukh, Y. M., Mohamed Abdelkarim AL-Ali, A. A., Adwan, A. O., Alhammadi, A., Shaikh, M. M., Faroukh, A. M., \& Femini, I. (2019). Environmental Monitoring using CanSat. 2019 6th International Conference on Space Science and Communication (IconSpace). https://doi.org/10.1109/ IconSpace.2019.8905942

Fernández, A., \& Medel, R. H. (2019). Estudio bibliográfico del estado del arte del desarrollo y aplicaciones educativas de cansats https://www.researchgate.net/ publication/332973924
Ferraz, M. C., Pereira, M. C., Greco, M., \& Peiró, E. (2017). Design of a Generic Platform for an Educational CanSat. In Proc. of the 1st IAA Latin American Symp. on Small Satellites.

Fretes, H., \& Gómez, R. (2018). Análisis e implementación de fotogrametría digital aplicada a imágenes aéreas | Fretes | Revista Científica de la Juventud. https://www.juventud. gov.py/ojs/index.php/snj1/article/view/17/16

Fuerza Aérea Colombiana. (2019). Fuerza Aérea trabaja en sinergia con Universidades de Antioquia para Lanzamiento de Cohetes. https://www.fac.mil.co/fuerza-aé rea-trabaja-en-sinergia-con-universidades-de-antioquiapara-lanzamiento-de-cohetes

Herrada, D. (2020). L3gd20lib. Available at https://github.com/ dafruit/Adafruit_L3GD20_U. Herrada, D. Lsm303lib.

Heyasa, B. L., \& Galarpe R. K., V. R. (2017). Initial Development and Testing of Microcontroller-MQ2 Gas Sensorfor University Air Quality Monitoring, 12(3), 47-53. https://doi. org/10.9790/1676-1203024753

Hunt, E. R., Doraiswamy, P. C., McMurtrey, J. E., Daughtry, C. S. T., Perry, E. M., \& Akhmedov, B. (2012). A visible band index for remote sensing leaf chlorophyll content at the Canopy scale. International Journal of Applied Earth Observation and Geoinformation, 21(1), 103-112. https:// doi.org/10.1016/j.jag.2012.07.020

INPE. (2020). Cubedesign. www.inpe.br/cubedesign/2020/es/

Ladyada. (2020). Bmp180lib. https://github.com/ adafruit/ Adafruit-BMP085-Library

Méndez, B. E. (2017). Diseño y construcción de un microsatelite (CanSat). España.

Mohd, R., Shariff, J., Kaushil, H., Likhita, S., Bhootpur Nikhil, \& Mohan Sreejith. (2020). Innovative Design, Analysis and Development Practices in Aerospace and Automotive Engineering (I-DAD 2018). Springer.

Ogata V., Contieri D., Silva L., Piñeroa, J., (2019). The CanSat project using Commercial-Off-The-Shelf components. https://www.researchgate.net/publication/340789283

Paulo Silva Neto, L., Victor, O. O., Danielle, C. P., Neto Lauro, S. P., \& Jhonathan, P. M. (2019). The CanSat project using Commercial-Off-The-Shelf components. https://www.re searchgate.net/publication/340789283

Seed. (2015). Grove-Gas Sensor (MQ2) User Manual. 